\title{
Cylindrical heterogeneous detonation waves
}

\author{
Z. GABRIJEL $\dagger$ AND J. A. NICHOLLS $\ddagger$ \\ Department of Aerospace Engineering, The University of Michigan, Ann Arbor, Michigan, U.S.A.
}

(Received 30 August 1977; revised 16 March 1978)

\begin{abstract}
Abatract-Further experimental studies of blast wave initiated cylindrical heterogeneous (liquid fuel drops, gas oxidizer) detonation waves are described. A pie-shaped shock tube, used for these studies, was altered in certain ways so as to improve the modeling of cylindrical waves. These modifications, along with some operational aspects, are briefly discussed. The breech of the facility, where the blast wave is generated by an explosive, became distorted with usage. Results are presented which show that lower detonation velocities are realized with the damaged breech (other conditions being the same). A photographic and pressure switch wave time of arrival study was made to ascertain the wave shape. Photographs are shown which show that the waves, blast as well as detonation, are close to cylindrical. However, in some cases there is appreciable distortion of the wave front by debris ahead of the wave. Presumably this debris comes from the blasting cap used to ignite the condensed explosive. A series of experiments was conducted using kerosene drops of $388 \mu \mathrm{m}$ diameter dispersed in air through use of a large number of hypodermic needles. Radial fuel void regions were established by cutting off the fuel flow to a number of needles. Preliminary results relating to the effect of the size of the cloud gap on detonation velocity, quenching, and the initiator energy levels required for detonation are discussed.
\end{abstract}

\section{Introduction}

THERE are many instances, accidentally as well as by choice, where unconfined homogeneous combustible clouds (all gaseous) or heterogeneous combustible clouds (liquid or solid fuel with gas oxidizer) are formed. In such cases, there is the possibility of complete deflagration of the cloud, transition to detonation, or the direct initiation of detonation by a high energy initiating source. Needless to say, there are many fundamental aspects that bear on these problems.

Personnel of this department have been interested and active in the study of the blast wave initiation of detonation for quite some time now. In line with this interest and in an attempt to maximize the level of understanding through a combined analytical-experimental study, an experimental facility was developed which models an idealized cylindrical cloud. The cloud is uniform except for possible variations in the radial direction. A special shock tube was designed and built which corresponds to a pie shaped sector of the cloud of a certain element of height. A number of investigations of the blast wave initiation of heterogeneous, as well as homogeneous, detonation were conducted and the results are available in the literature. $\$$

Experience and the requirement for better definition of the results indicated the need for some modifications to the facility. These modifications were made

†Doctoral Candidate.

$\ddagger$ Professor.

§See Fry and Nicholls (1974a), Fry and Nicholls (1974b), and Nicholls et al. (1974). 
and are briefly described herein. A follow-on section presents results showing the importance of the breech condition to the strength of the blast waves and heterogeneous detonation waves produced. Also, the results of a study on the shape of the blast waves and detonation waves generated is presented. Finally, the results of heterogeneous detonation tests wherein a fuel void region of variable radial extent exists is presented and discussed.

\section{Experimental facility}

The initial sectored shock tube was $72.90 \mathrm{~cm}$ in radius, $20^{\circ}$ included angle, and $5.08 \mathrm{~cm}$ between the side walls (cloud height), as can be seen in Fig. 1. The heterogeneous cloud is produced by flowing the liquid fuel to a number of capillary needles and disturbing this flow at the Rayleigh frequency, thus forming uniform size drops. The cylindrical blast wave is produced by means of a blasting cap and controlled amounts of the condensed explosive, Detasheet, in the breech. The wave position as a function of time is sensed by a number of pressure switches, with their output conducted to a raster circuit in an oscilloscope.

In the earlier experiments, the radial position where the blast wave began to undergo transition to detonation was usually about $2 / 3$ of the chamber maximum radius. This precluded a detailed study of the transition zone and hence some important aspects of the blast wave initiation of detonation. Consequently, the accurate prediction of threshold initiation energies was questionable. In view of this limitation, an extension of the chamber was designed and built. In adding the extension, the $20^{\circ}$ angle and $5.08 \mathrm{~cm}$ wall spacing were retained but the chamber's radial size increased to $137.67 \mathrm{~cm}$. This necessitated a number of changes of the original facility. The main changes are outlined below. A more detailed description of these, and other changes, are described elsewhere (Nicholls et al.,

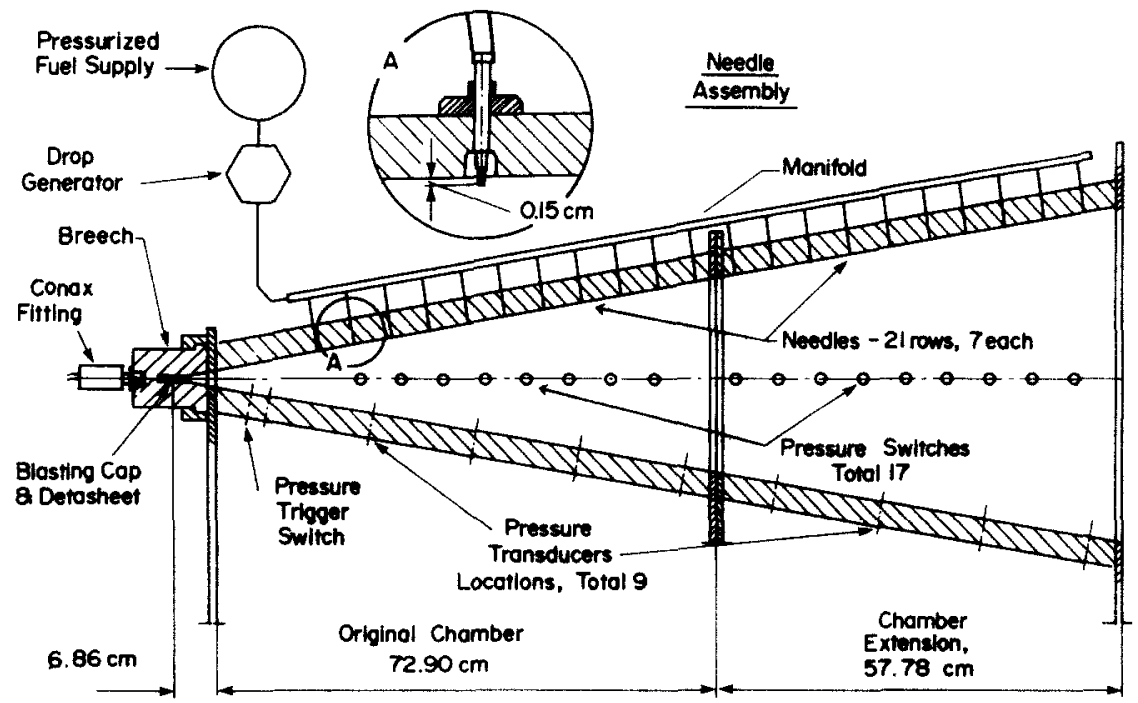

Fig. 1. Schematic of experimental arrangement. 
1976). The greater radial size of the chamber demanded enlargement of the fuel manifold as well as an increase in the number of submanifolds and needles. The number of fuel needles increased from 77 (11 rows with 7 per row) to 147 (21 rows with 7 per row). Also, nine additional pressure switches were installed. These changes are shown in Fig. 1.

The production of drops of uniform size and spacing from 147 needles was a tedious and time consuming process. Wetting of the upper and lower walls was particularly troublesome. Wetting of the upper wall was solved by projecting the needles slightly into the chamber, as shown in Insert A of Fig. 1. Without this projection, surface tension, combined with the initial inclination of the needles, caused the cavity to fill with liquid and liquid fuel to flow along the inclined upper wall. Occasionally, this flow would spread to the side walls of the chamber. Wetting of the bottom wall was partially solved by shortening, as much as possible, the interval between retraction of the absorbent fuel pad on the bottom wall and activation of the initiator charge. On occasion, the side walls would become wetted. Invariably this was attributable to damaged needle tips, which were then replaced.

\section{Experimental results}

\section{Blast wave generation-damaged breech}

In this facility the blast wave is produced by means of a blasting cap and selected amounts of the condensed explosive, Detasheet C (63\% PETN-8\% NC, a Dupont product) which are located in the breech. The breech is designed to give a smooth transition from a small cylindrical cavity to the main part of the chamber. It is well known that the details of the initiating source has a pronounced effect on the properties of the blast wave produced-at least for small radii. Non-uniformities in explosive loading, unsteady wave development and the shape of the breech cavity were probably, among others, the main factors that affected characteristics (i.e. strength and uniformity) of the wave produced.

The extreme thermal and mechanical stresses encountered in the cavity led to significant deformation of the cavity's contour. It was observed that the deformations of the breech cavity were different depending on the type of mixture used. The distortion of the cavity in the breech which was, for the most part, used in gaseous detonation studies showed that its cylindrical shape was mostly preserved although increased about fivefold in size. The breech that was exclusively used for heterogeneous detonation studies had the cavity distorted in a different fashion. The cavity was deformed towards a spherical shape around the center of the explosion. Ultimately, the size of this cavity was about 3-5 that of the initial cavity. In both cases the cavity's surfaces were strongly corrugated. The difference in the cavity's deformation must be due, in part, to the fact that in the heterogeneous detonation studies there is no combustible mixture in the breech when the initiator is activated. That was not the case when a gaseous mixture was used. In the course of the experiments, it was observed that the enlargement of the cavity, after an initial fast increment, decelerated consider- 
ably. After approximately 100 experimental runs, the volume of the cavity appeared to remain constant.

The damaged breech had a definite effect on the blast wave produced. For any radius and for a given initiator energy, the strength of the blast wave was less than in the case of a good breech. Further, initiator energy levels that had produced detonation in the past were now insufficient; higher threshold energy levels were required. An attempt was made to salvage one of the damaged breeches through use of a machined insert. This was helpful but the insert soon failed. Accordingly, a new breech was made with some improvements incorporated (Nicholls et al., 1976).

The results of a large number of experiments on blast initiated detonation are illustrated in Fig. 2, which shows the ranges of the experimental results obtained with the new and damaged breech. These results are for a uniformly dispersed heterogeneous mixture. In this figure, the radius $r$, and the time, $t^{\prime}$, are measured from the apex of the chamber and the trigger switch, respectively. $\dagger$ The malfunction of the pressure switches was easy to detect as can be seen from Fig. 2 , damaged breech curves, near $110 \mathrm{~cm}$. It is apparent that the new breech made a significant improvement. In all of the cases when a new breech was used, a heterogeneous detonation was clearly established at a much greater radial position. On the radius vs time plots a detonation is significant by a straight line, that is, a constant velocity wave.

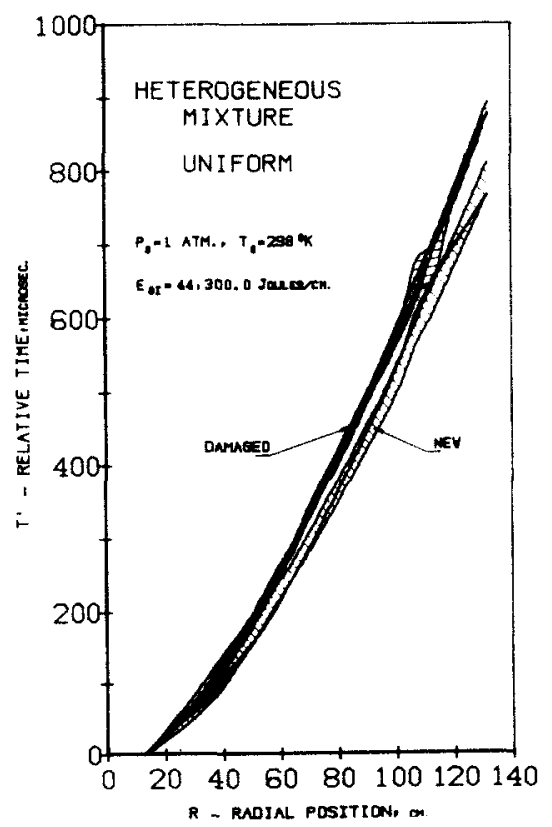

Fig. 2. Range of the experimental data for damaged and new breech, $E_{o i}=44,300 \mathrm{~J} / \mathrm{cm}$.

$\dagger$ The computer graphic plots were restricted to capital letters such as $R, T^{\prime}, P_{O}$, and $E_{O I}$ in place of the more conventional $r, t^{\prime}, p_{o}$, and $E_{o i}$. 


\section{Shape of the wave front}

Inasmuch as the experimental facility was designed to model cylindrical heterogeneous detonation waves, some studies were directed at determining the actual shape of the waves. This investigation was performed using two different experimental techniques. First, a new arrangement of the pressure switches was employed. Some were left on the centerline of the side wall, as before, some were located along the bottom, and others were located on the side wall very close to the bottom. Thus, differences in wave time of arrival at the various positions gave an indication of wave shape. In addition, instantaneous shadowgraph photographs of the wave as it emerged from the exit of the chamber (before strong three-dimensional effects were realized) were taken. Due to the greater size of the chamber exit $(48.26 \mathrm{~cm})$ as compared to the diameter of the mirrors $(30.48 \mathrm{~cm})$, separate photographs of the upper and lower halves of the emerging wave were taken. Each photograph presented herein shows a different experimental run.

The rearranged pressure switch experiments indicated that the shape of the wave front (blast wave as well as detonation) usually approximated the ideal cylindrical shape. However, it was found that the actual shape of the wave front was not always ideal. In such cases the greatest departures from the ideal shape have been found to exist within the extended part of the chamber. The exact cause is unknown yet. The probable causes could be growth of the boundary layer on the side walls, possible wave structure in the flow behind a leading wave and a non-ideal blast wave development, part of which could be attributed to the damaged breech.

Representative photographs of the wave emerging from the chamber for the case of calculated initiator blast energy level $E_{o i}=44,300 \mathrm{~J} / \mathrm{cm}$ are shown in Fig. 3. Each photograph has labels showing the run number, time delay from the triggering switch, an arrow showing the centerline of the chamber, and an arrow showing the wave direction. The ideal cylindrical wave drawn on the photographs is shown as a chopped line right at the exit. The cylindrical shape corresponding to the actual wave position (on the chamber centerline) is shown as a dotted line. It can be seen that the wave shape is very close to ideal in the cases when a wave front was right at the exit. This is also in agreement with the pressure switch data. The prominent distortions of the wave front, seen in the figure, occurred frequently and are presumably due to debris from the blasting cap. This debris was traveling supersonically and, hence, there are associated conical shock waves. This debris was noted more frequently when low initiator energies were used. It is believed that the debris, in many cases, overtook the blast wave as it slowed down. The waves associated with debris, or debris itself, could cause premature firing of the pressure switches.

In general, the shape of the detonation wave was not quite as close to cylindrical as in the case of blast waves, and there was some indication that the wave was somewhat slower in the upper half. This, of course, could be due to the cavities for the needles in the top wall, the protruding needle tips, and the fact that the liquid fuel jets would not be broken up right at the exit of the needles. In some runs considerable debris was observed, as can be seen in Fig. 4. In such cases there was considerable scatter in the radius-time data. 


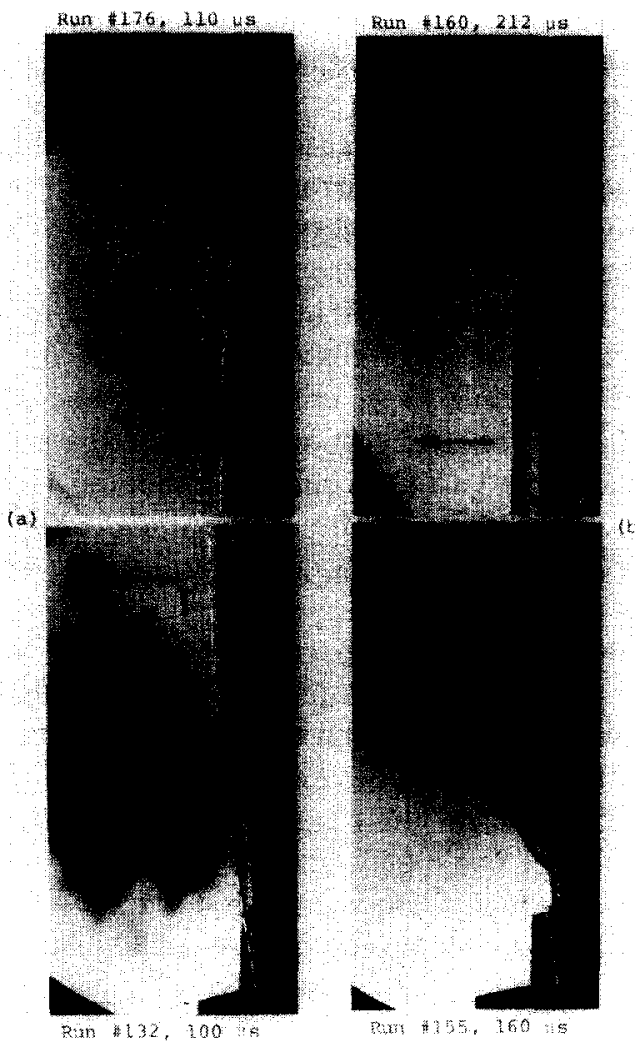

Fig. 3. Photographs of wave front at the chamber exit, $E_{o i}=44,300 \mathrm{~J} / \mathrm{cm}$. (a) Blast wave front. (b) Heterogeneous wave front.

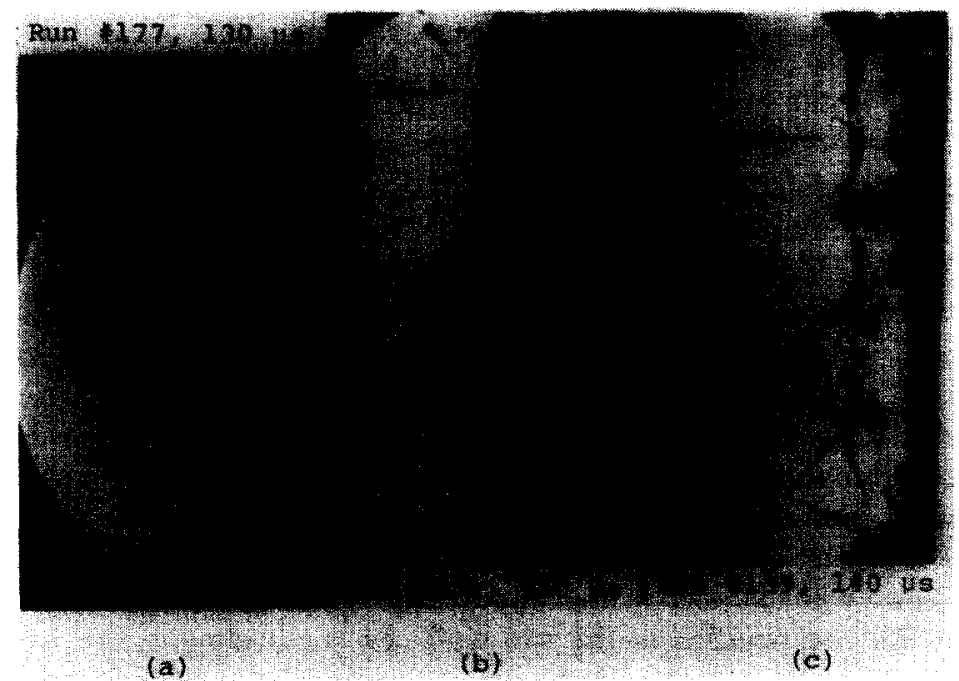

Fig. 4. Photographs of wave front at the chamber exit, $E_{0 i}=27,250 \mathrm{~J} / \mathrm{cm}$. (a) and (c) Blast wave front. (b) Heterogeneous wave front. 


\section{Detonation through a non-uniform heterogeneous mixture}

Before investigating those situations wherein the mixture ratio would vary with radius, it was desired to conduct a series of experiments with uniform heterogeneous mixtures. Such experiments had not been performed in the extended chamber and it was logical to use such data for basic-reference purposes. Hence, a number of experiments were conducted. Kerosene drops of $388 \mu \mathrm{m}$ diameter, a mixture ratio of $\phi=0.753$, and air at room temperature and pressure were used. The first and 21st (last) needle rows are located at a radial distance of $24.60 \mathrm{~cm}$ and $134.40 \mathrm{~cm}$ respectively. The rows in between are evenly distributed along the top wall of the chamber with the radial increment, $\Delta r=$ $5.48 \mathrm{~cm}$. The initiator energy levels, $E_{o i}$, were varied over a wide range during the course of experimental research.

Discrete points for one experiment using $E_{o l}=52,900 \mathrm{~J} / \mathrm{cm}$ are shown in Fig. 5 along with the radius-time curve. This figure is typical; the experimental results showing high consistency and relatively small scatter. For initiator energies, usually corresponding to $E_{o i}=30,650 \mathrm{~J} / \mathrm{cm}$ or more, but occasionally for $E_{o i}=27,280 \mathrm{~J} / \mathrm{cm}$, detonation was achieved. Careful study of this figure indicates that a constant velocity detonation (straight line on the $r-t^{\prime}$ curve) is apparently established at about $60 \mathrm{~cm}$ and extending to about $90 \mathrm{~cm}$ with a Mach number of about 4.3 . However, at about $95 \mathrm{~cm}$, a second lower constant velocity wave (Mach number about 3.2) is noted which is maintained to the exit of the chamber. The calculated Mach number of $C-J$ detonation is found to be 5.06. This dual velocity nature was observed in many of the higher energy level runs with the second transition point commonly occurring near $100 \mathrm{~cm}$. This radius is well into the new portion of the chamber. The reason for this behaviour is not

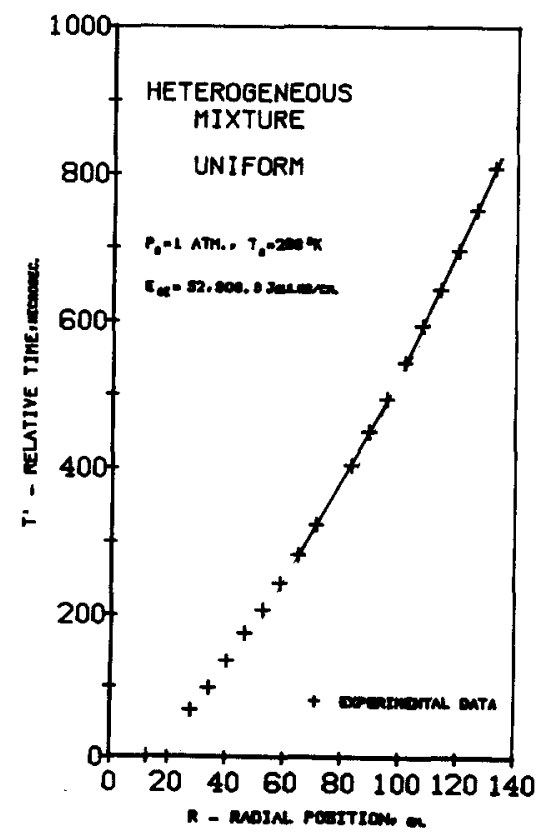

Fig. 5. Experimental uniform heterogeneous data, $r$ vs $t^{\prime}, E_{o l}=52,900 \mathrm{~J} / \mathrm{cm}$. 
known with certainty but boundary layer growth on the side walls is suspected and is under investigation. The results of many other experiments in the extended chamber are presented elsewhere (Nicholls et al., Phase II, 1976).

It was of interest to investigate cases where a non-uniform heterogeneous mixture was deliberately established. Two aspects of particular interest were the following:

1. To what extent would a localized void in fuel affect propagation of a heterogeneous detonation wave and how great (spacially) a void would be required to terminate the detonation?

2. What would be the minimum blast wave initiator strength necessary to institute a heterogeneous detonation under non-uniform conditions?

In order to create the non-uniform distribution, some of the needles were removed from the chamber and the fuel from these needles channeled into a separate container. This was necessary (in contrast to simply blocking the needles) in order to maintain good drop production for the rest of the needles in use. The holes in the top of the chamber left by the removed needles were filled with solid rods.

The void in fuel, as shown in Fig. 6, was chosen as a region near the theoretical critical radius, $r^{*}$, where the blast energy and energy of combustion contained within $r^{*}$ are equal. It is around this radius that the transition from blast wave to detonation occurs and it was of interest to determine the sensitivity of the transition process to the extent of the fuel void region. Under these conditions, the variation in equivalence ratio along the chamber is characterized

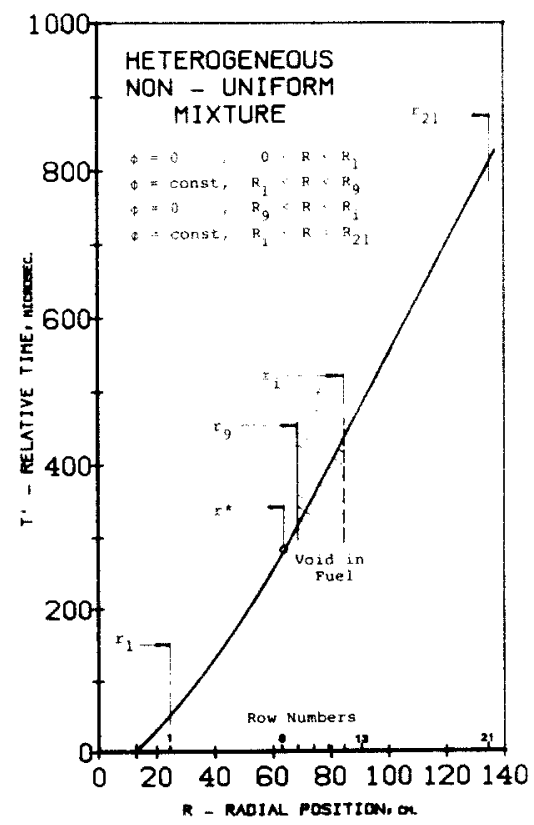

Fig. 6. Non-uniform heterogeneous arrangement. 
as follows:

and

$$
\begin{array}{llr}
\phi=0 & \text { for } & r<r_{1} \\
\phi=0.753=\text { const } & \text { for } & r_{1}<r<r_{9} \\
\phi=0 & \text { for } & r_{9}<r<r_{i}
\end{array}
$$

$$
\phi=0.753=\text { const for } r_{i}<r<r_{21}
$$

The radial position of the needle row 9 is $r_{9}=68.40 \mathrm{~cm}$. There is no fuel for any condition before $r_{1}$. The position of the last removed row in a particular experimental run is denoted by $r_{i}$.

A number of experiments were conducted wherein consecutive needle rows 9-12 were removed. The size of the fuel void region (in the radial direction) was $10.9 \mathrm{~cm}, 16.5 \mathrm{~cm}, 21.8 \mathrm{~cm}$ and $27.4 \mathrm{~cm}$, respectively. The initial blast energy ranges from 27,250 to $44,300 \mathrm{~J} / \mathrm{cm}$. These energy levels correspond to the energy content in a blasting cap plus the amount of condensed explosive from 1.75 to $3.0 \mathrm{~g}$.

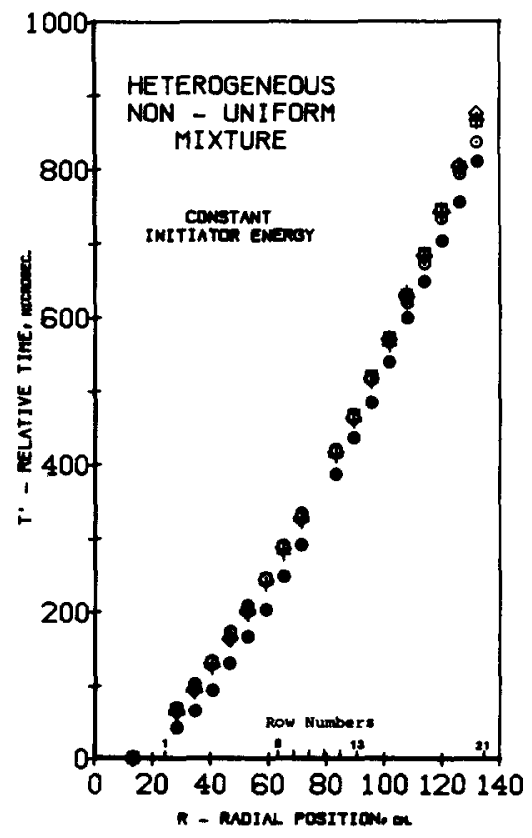

Fig. 7.

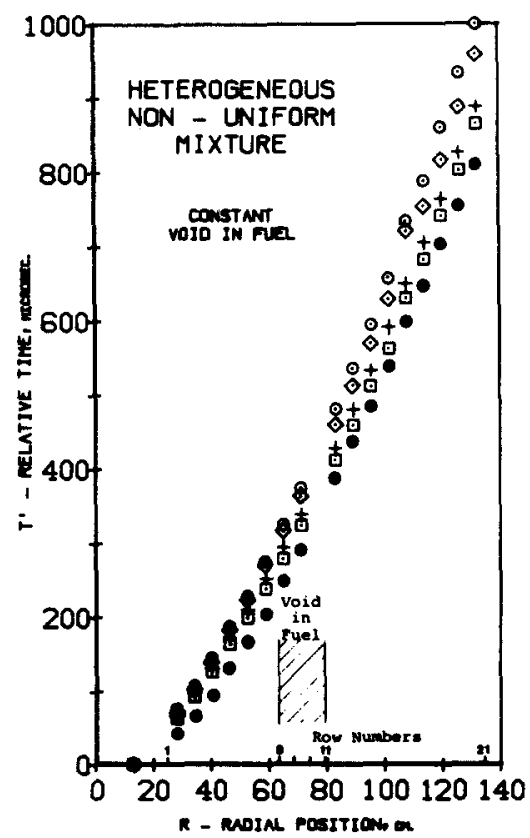

Fig. 8.

Fig. 7. Experimental non-uniform heterogeneous data, $r$ vs $t^{\prime}, E_{o}=44,300 \mathrm{~J} / \mathrm{cm}$. , Uniform; $\square$, row 9 removed; +, rows 9 and 10 removed; $\diamond$, rows $9-11$ removed; and $O$, rows $9-12$ removed.

Fig. 8. Experimental non-uniform heterogeneous data, $r$ vs $t^{\prime}$, constant void in fuel $=$ $16.5 \mathrm{~cm}$ (rows 9 and $10 \mathrm{removed}$ ). $, E_{\omega}=4,300 \mathrm{~J} / \mathrm{cm}$, uniform; $\square, E_{o l}=44,300 \mathrm{~J} / \mathrm{cm}$; $+, E_{d i}=37,500 \mathrm{~J} / \mathrm{cm} ; \circlearrowleft, E_{o t}=30,650 \mathrm{~J} / \mathrm{cm}$; and $O, E_{o l}=27,250 \mathrm{~J} / \mathrm{cm}$. 
The obtained experimental results, radius versus time, are shown in Figs. 7 and 8. For each case presented, only one characteristic run is given. This selection was adopted to avoid obscuring the data. Figure 7 illustrates the influence of the number of needle rows removed for a given initiator energy level which was, in this case, high, $E_{\mathrm{o} i}=44,300 \mathrm{~J} / \mathrm{cm}$. Figure 8 illustrates the influence of initiator energy level for a fixed size of the fuel void region. A moderate void in fuel was chosen and it was equal to $16.5 \mathrm{~cm}$.

Referring to Fig. 7, the constant initiator energy case, the uniform cloud case shows the two distinct velocities characteristic, as mentioned earlier. For this run the Mach number were somewhat higher, yielding values of Mach numbers of 4.58 and 3.36. With the removal of one or two needle rows (void up to $16.5 \mathrm{~cm}$ ), no appreciable differences were noted and the two measured velocities were very close to the uniform case values. However, when the third needle row was removed (void $=21.8 \mathrm{~cm}$ ), only one detonation velocity, the second slower one, was observed and the value was close to the previous cases. An anomaly appeared when removal of a fourth row (void $=27.4 \mathrm{~cm}$ ) led to the two detonation velocity result again with the first velocity being somewhat smaller than in the smallest void case but the second velocity being as large or larger. This result was unexpected and at this point is unexplained.

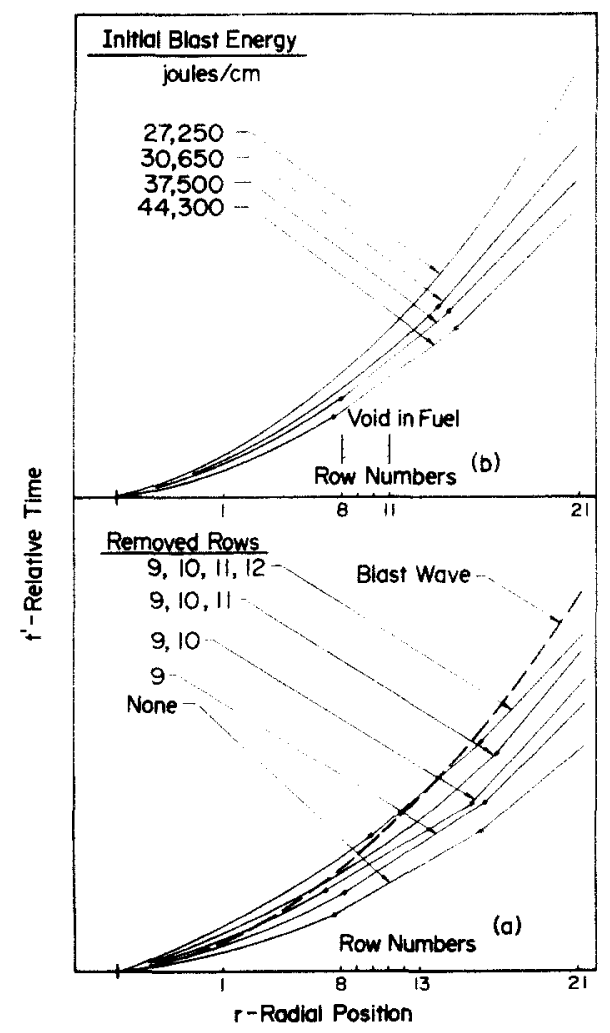

Fig. 9. Quantitative trend of non-uniform heterogeneous detonation wave propagation. (a) $E_{o i}$ constant; void in fuel varied. (b) Void in fuel constant; $E_{o i}$ varied. 
For the case shown in Fig. 8, constant void in fuel, the wave propagation characteristics are much clearer. The highest initiator energy used produced two distinct detonation velocities with characteristics similar to those discussed previously. With a decrease in initiator energy (to a moderate initiator energy level) only one detonation velocity was observed. The transition of a blast wave to this detonation velocity occurred at a radial position of about $90 \mathrm{~cm}$ and the constant velocity was maintained until the chamber exit. The lowest initiator energy used produced no detonation and the wave monotonically decayed in velocity and strength.

In general, a decrease in initiator energy level with increased void in fuel resulted in lower detonation velocities or no detonation at all. The scatter in experimental results was small. For the cases presented in Figs. 7 and 8, the scatter in data was below or equal to $15 \mu$ secs (for all energy levels and voids in fuel investigated) measured at the position of the last pressure switch ( $r=$ $131.57 \mathrm{~cm}$ ). At the smaller radii the observed scatter was even smaller. Figure 9 shows in qualitative fashion the behavior of the waves investigated and discussed herein. The dots on the curves represent the beginning and the end of constant velocity regions.

The foregoing results on the propagation of heterogeneous detonation through a non-uniform mixture must be regarded as preliminary. Certainly more experiments and accompanying analysis are needed. There is the possibility that, given an even greater radius tube, the detonation would not continue at the second relatively low Mach number. It could possibly attenuate or even accelerate. Attention should be, and is, given to the side wall boundary layers.

Acknowledgement - This research was supported in part by Eglin Air Force Base under Contract No. F08635-74-C-0123 and Army Research Organization Grant DAAG29-77-G-0104.

\section{References}

Fry R. S. and Nicholls J. A. (1974a) Blast initiation and propagation of cylindrical detonations in MAAP-Air mixtures. AIAA J. 12, 1703-1708.

Fry R. S. and Nicholls J. A. (1974b) Blast wave initiation of gaseous and heterogeneous cylindrical detonation waves. 15th Symp. (Int.) on Combustion, The Combustion Institute, Pittsburgh, Pennsylvania.

Nicholls J. A., Sichel M., Fry R. and Glass D. R. (1974) Theoretical and experimental study of cylindrical shock and heterogeneous detonation waves. Acta Astronautica 1, 385-404.

Nicholls J. A., Sichel M., Fry R. S., Oza M. and Gabrijel Z. (1976) Fundamental aspects of unconfined explosions: Phase I. AFATL-TR-76-77.

Nicholls J. A., Sichel M., Gabrijel Z. and Oza R. (1976) Fundamental aspects of unconfined explosions: Phase II. AFATL-TR-76-78. 